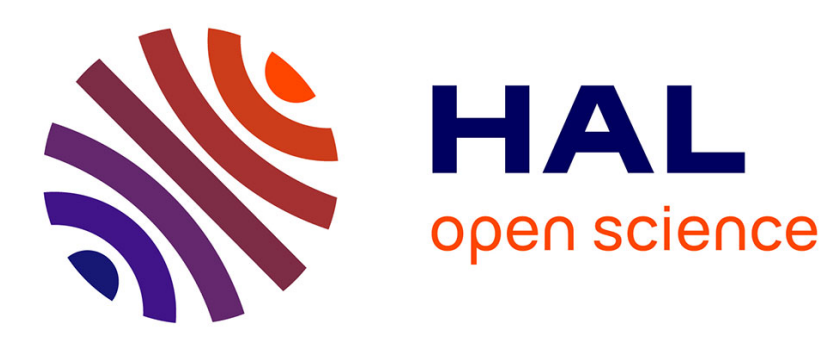

\title{
On first range times of linear diffusions
}

Paavo Salminen, Pierre P. Vallois

\section{To cite this version:}

Paavo Salminen, Pierre P. Vallois. On first range times of linear diffusions. Journal of Theoretical Probability, 2005, 18 (3), pp.567-593. hal-00141268

\section{HAL Id: hal-00141268 https://hal.science/hal-00141268}

Submitted on 13 Apr 2007

HAL is a multi-disciplinary open access archive for the deposit and dissemination of scientific research documents, whether they are published or not. The documents may come from teaching and research institutions in France or abroad, or from public or private research centers.
L'archive ouverte pluridisciplinaire HAL, est destinée au dépôt et à la diffusion de documents scientifiques de niveau recherche, publiés ou non, émanant des établissements d'enseignement et de recherche français ou étrangers, des laboratoires publics ou privés. 


\title{
On first range times of linear diffusions
}

\author{
Paavo Salminen \\ Åbo Akademi University \\ Mathematical Department \\ FIN-20500 Åbo, Finland \\ email: phsalmin@abo.fi
}

\author{
Pierre Vallois \\ Université Henri Poincaré \\ Département de Mathématique \\ F-54506 Vandoeuvre les Nancy, France \\ email: vallois@iecn.u-nancy.fr
}

May 26, 2006

\begin{abstract}
In this paper we consider first range times (with randomised range level) of a linear diffusion on $\mathbf{R}$. Inspired by the observation that the exponentially randomised range time has the same law as a similarly randomised first exit time from an interval, we study a large family of nonnegative 2-dimensional random variables $\left(X, X^{\prime}\right)$ with this property. The defining feature of the family is $F^{c}(x, y)=F^{c}(x+y, 0), \quad \forall x \geq$ $0, y \geq 0$, where $F^{c}(x, y):=\mathbf{P}\left(X>x, X^{\prime}>y\right)$.

We also explain the Markovian structure of the Brownian local time process when stopped at an exponentially randomised first range time. It is seen that squared Bessel processes with drift are serving hereby as a Markovian element.
\end{abstract}

Keywords: Bessel bridges, Bessel functions, Brownian motion, $h$-transforms, infimum, Ray-Knight theorem, supremum. 


\section{Introduction}

One aim of this paper is to increase understanding of the interplay between the first range time and the first exit time from an interval for a linear diffusion with state space $\mathbf{R}$. Our main result in this direction is Proposition 3.3 which generalizes Théorème 4 in Vallois [32]. To explain the result shortly, consider a Brownian motion $\left\{W_{t}: t \geq 0\right\}, W_{0}=0$, and define

$$
S_{t}:=\sup _{0 \leq s \leq t} W_{s} \quad \text { and } \quad I_{t}:=-\inf _{0 \leq s \leq t} W_{s}
$$

Introduce also a random variable $U$ which is uniformly distributed on $(0, a)$, $a>0$, and independent of the Brownian motion. Then it is proved in [32] (see also Vallois [33] for further developments) the Brownian motion stopped when $S_{t}+I_{t}$ exceeds $U$ for the first time is identical in law with the Brownian motion stopped when it exits the interval $(U, U-a)$.

The result in Proposition 3.3 below states that there exists a large class of random variables $\left(X, X^{\prime}\right)$ which can be used instead of $(U, a-U)$ so that the identity in law remains valid. In particular, we may take $X$ and $X^{\prime}$ to be independent and identically exponentially distributed random variables. It is also seen that the result is valid for all diffusions on $\mathbf{R}$.

The starting point of our study was not, however, the desire to generalize the result in [32] but the observation that the two seemingly different formulae in Borodin and Salminen [5] are, in fact, identical. Indeed, the Laplace transforms in No. VI.14 p. 112 and No. VI.23 p. 118 in [5] (for the latter formula, see Corollary 4.5 below) are the same when in the first one we take $\beta=\alpha$. This can be proved directly, but it is also seen to be an immediate consequence of our main identity in law, see Section 4.

The formulae mentioned above concern the joint distribution of local times on different levels of Brownian motion stopped at the first exit time from an interval and stopped at the first range time, respectively. The results in No. VI.14 and No. VI.23 are due to Borodin, see [3] and [4], where, however, the results are presented in another form. These formulae are derived via the Ray-Knight theorems presented, e.g., in No. V.8 and No. V.7 in [5], respectively. We refer also to Vallois [31] for further generalizations of the Ray-Knight theorems.

Taking Laplace transforms means that we randomise, using exponential variables, on the one hand, the length of the interval and, on the other hand, the range width. The simple and nice structure of, especially, the formula 
in No. VI.23 and the surprising cancellations when deriving it made us to seek a generalisation of the formula by lifting it to the level of processes. As a result, it is seen that the local time of Brownian motion stopped at randomised range time and considered as a process in space parameter is a Markov process expressed in terms of squared Bessel processes of dimensions 2 and 4 with drift. By the main identity in law, Proposition 3.3, the local time process of Brownian motion stopped at randomised first exit time has the same description. We remark that the Brownian motion stopped at these random times is not Markovian.

Range and the first range time of a stochastic process are well studied objects. For fixed time and asymptotical results in discrete time concerning the range, see, for instance, Dvoretsky and Erdös [9], Feller [10], Jain and Orey [18], Jain and Pruitt [19], Chosid and Isaac [7], [8], Glynn [12], and Vallois [34]. The distribution of the range of Brownian motion was computed by Feller [10], see also Imhof [16], especially, for the Laplace transform of the first range time. Other works in continuous time are, e.g., Hsu and March [15], Imhof [17], Pitman and Yor [23], and Cheng, Cowan and Holst [6]. For some applications, see Troutman [29], Hooghiemstra [14], and Tapiero and Vallois [28].

The paper is organised so that in the next section we present and analyse the class of 2-dimensional random variables which are used to randomise the length of the interval associated with the first exit time. In Section 3 we discuss the main identity in law. In Section 4 we present our Ray-Knight theorem for Brownian motion stopped at randomised range time. Further, using this theorem we give a new proof of the result in No. VI.23 p. 118 in [5], which explains well the structure of the result.

\section{The class $\mathcal{K}$ of random variables}

In this section we analyse a class of 2-dimensional random variables used in the next section to randomise the first range time and the first exit time from an interval for a diffusion. The basic property we are after in this respect is that these random times should be identical in law. Roughly speaking, this class consists of mixtures of uniformly distributed variables. Our exact definition is as follows

Definition 2.1 $\mathcal{K}$ is the set of 2 -dimensional positive random variables 
$\left(X, X^{\prime}\right)$ such that

$$
\left(X, X^{\prime}\right) \stackrel{(\mathrm{d})}{=}(U V,(1-U) V),
$$

where the random variable $U$ is uniformly distributed on $[0,1]$ and $V$ is an arbitrary positive random variable independent of $U$.

Remark 2.2 a) The class $\mathcal{K}$ can also be constructed by taking

$$
\left(X, X^{\prime}\right) \stackrel{(\mathrm{d})}{=}(\tilde{U}, \tilde{V}-\tilde{U}),
$$

where $\tilde{U}$ is uniformly distributed on $[0, \tilde{V}]$ and $\tilde{V}$ is an arbitrary positive random variable.

b) Notice that if $\left(X, X^{\prime}\right) \in \mathcal{K}$ then $X+X^{\prime}$ and $X /\left(X+X^{\prime}\right)$ are independent. Moreover,

$$
X+X^{\prime} \stackrel{(\mathrm{d})}{=} V, \quad \text { and } \quad \frac{X}{X+X^{\prime}} \stackrel{(\mathrm{d})}{=} U .
$$

Definition 2.3 $\mathcal{M}$ is the set of measures $\nu$ on $\mathbf{R}_{+}$such that

$$
\int_{0}^{\infty} v \nu(d v)=1 .
$$

Below we use the notation $F_{X}$ for the distribution function of a random variable $X$. If the derivative $F_{X}^{\prime}$ exists almost everywhere we say that $X$ has a density, and the density is then taken to be equal to $F_{X}^{\prime}$.

In the next proposition we give a characterization of the class $\mathcal{K}$ from which many distributional properties can be derived. Another characterization, which is used in Section 3, is presented in Proposition 2.6.

Proposition 2.4 The non-negative random variable $\left(X, X^{\prime}\right)$ is in $\mathcal{K}$ if and only if

$$
\mathbf{P}\left(X \in d a, X^{\prime} \in d b\right)=d a \pi(a, d b)=d b \pi(b, d a), \quad a>0, b>0,
$$

where $\pi(a, \cdot)$ is for every $a>0$ a positive and finite measure on $\mathbf{R}_{+}$representable for all $a \geq 0$ in terms of a measure $\nu \in \mathcal{M}$ via

$$
\pi(a, B)=\nu(a+B), \quad a+B:=\{a+b: b \in B\},
$$

where $B$ is a Borel set in $\mathbf{R}_{+}$, in other words, $\pi(a, d b)$ is for every $a \in \mathbf{R}_{+}$ the image of $\nu(d v), v \geq a$, under the mapping $x \mapsto x-a$. Moreover, if 
$\left(X, X^{\prime}\right) \in \mathcal{K}$ then $X$ and $X^{\prime}$ are identical in law, the density of $X$ exists, and is given by

$$
F_{X}^{\prime}(x)=\nu([x, \infty))=\int_{[x, \infty)} v^{-1} F_{V}(d v),
$$

where $F_{V}(d v)=v \nu(d v)$ determines the distribution of $V=X+X^{\prime}$ associated with $\left(X, X^{\prime}\right)$.

Proof Assume $\left(X, X^{\prime}\right) \in \mathcal{K}$, and let $F_{V}$ denote the distribution of $V$. Then

$$
\begin{aligned}
\mathbf{E}\left(\varphi\left(X, X^{\prime}\right)\right) & =\mathbf{E}(\varphi(U V,(1-U) V)) \\
& =\int_{\mathbf{R}_{+}^{2}} \varphi(u v,(1-u) v) \mathbf{1}_{\{u \leq 1\}} d u F_{V}(d v) .
\end{aligned}
$$

Introducing new integration variables $a=u v$ and $b=(1-u) v$ gives

$$
\mathbf{E}\left(\varphi\left(X, X^{\prime}\right)\right)=\int_{\mathbf{R}_{+}^{2}} \varphi(a, b) d a \pi(a, d b),
$$

where

$$
\pi(a, d b):=(a+b)^{-1} F_{V}(a+d b) .
$$

This relation defines the measure

$$
\nu(d v)=v^{-1} F_{V}(d v),
$$

and, conversely, $F_{V}(d v)=v \nu(d v)$. It is clear that $\nu \in \mathcal{M}$ because $F_{V}$ is a distribution function.

Let $\left(X, X^{\prime}\right)$ a 2-dimensional random variable satisfying (1). It is easily seen that the law of the 2-dimensional random variable $\left(\frac{X}{X+X^{\prime}}, X+X^{\prime}\right)$ is $1_{\{u \leq 1\}} v d u \nu(d v)$ and then, putting $\frac{X}{X+X^{\prime}}=U$ and $X+X^{\prime}=V$, we see that the random variables $U$ and $V$ are independent and $U$ is uniformly distributed on $[0,1]$. As a result, $\left(X, X^{\prime}\right) \in \mathcal{K}$.

The claim (2) concerning the density of $X$ follows immediately from (1) and the identification of the measure $\nu$ given in (3).

It is practical to specify the contents of Proposition 2.4 to the case when $\left(X, X^{\prime}\right)$ has a density. 
Corollary 2.5 Let $\left(X, X^{\prime}\right)$ be a 2-dimensional random variable.

1. Suppose there exists a measurable function $(a, b) \mapsto \pi(a, b)$ such that

$$
\mathbf{P}\left(X \in d a, X^{\prime} \in d b\right)=\pi(a, b) d a d b, \quad a>0, b>0 .
$$

Then $\left(X, X^{\prime}\right) \in \mathcal{K}$ if and only if there exists a function $\rho$ satisfying

$$
\int_{0}^{\infty} v \rho(v) d v=1
$$

such that for almost everywhere in $\mathbf{R}_{+}^{2}$

$$
\pi(a, b)=\rho(a+b)
$$

2. Suppose $\left(X, X^{\prime}\right) \in \mathcal{K}$. Then $\left(X, X^{\prime}\right)$ has a density if and only if the corresponding variable $V$ has a density. In this case, the latter is given by

$$
F_{V}^{\prime}(v)=v \rho(v), \quad v>0 . "
$$

The next result gives a clean and very useful characterization of the class $\mathcal{K}$ in terms of the distribution function of $\left(X, X^{\prime}\right)$.

Proposition 2.6 Let $\left(X, X^{\prime}\right)$ be a 2-dimensional non-negative random variable. Then

$$
\left(X, X^{\prime}\right) \in \mathcal{K} \quad \Leftrightarrow \quad F^{c}(x, y)=F^{c}(x+y, 0),
$$

where for $x>0, y>0$

$$
F^{c}(x, y):=\mathbf{P}\left(X>x, X^{\prime}>y\right)
$$

Proof Assume that $\left(X, X^{\prime}\right) \in \mathcal{K}$. From (1) we obtain for $x, y>0$

$$
\begin{aligned}
\mathbf{P}\left(X>x, X^{\prime}>y\right) & =\int_{x}^{\infty} d a \int_{(y, \infty)} \pi(a, d b) \\
& =\int_{x}^{\infty} d a \int_{(a+y, \infty)} \nu(d u) \\
& =\int_{(x+y, \infty)} \nu(d u) \int_{x}^{u-y} d a \\
& =\int_{(x+y, \infty)}^{u \nu(d u)-(x+y) \nu((x+y, \infty))}
\end{aligned}
$$


proving that $(x, y) \mapsto F^{c}(x, y)$ is a function of the sole variable $x+y$ and then $F^{c}(x, y)=F^{c}(x+y, 0)$.

To prove the opposite implication, consider for $\Delta x>0$

$$
\begin{aligned}
0 & \leq \mathbf{P}\left(x<X<x+\Delta x, x<X^{\prime}<x+\Delta x\right) \\
& =F^{c}(x, x)-F^{c}(x+\Delta x, x)-F^{c}(x, x+\Delta x)+F^{c}(x+\Delta x, x+\Delta x) \\
& =F^{c}(2 x, 0)-2 F^{c}(2 x+\Delta x, 0)+F^{c}(2 x+2 \Delta x, 0) .
\end{aligned}
$$

Consequently,

$$
\frac{1}{2}\left(F^{c}(2 x, 0)+F^{c}(2 x+2 \Delta x, 0)\right) \geq F^{c}(2 x+\Delta x, 0) .
$$

It follows from (4) that $x \mapsto F^{c}(x, 0)$ is convex. Therefore, its second derivative exists as a measure. Letting $\nu_{F}$ denote this measure we have from the definition of $F^{c}$

$$
\mathbf{P}\left(X \in d a, X^{\prime} \in d b\right)=d a \nu_{F}(a+d b), \quad a>0, b>0
$$

and, hence, by Proposition 2.4, $\left(X, X^{\prime}\right) \in \mathcal{K}$.

Corollary 2.5 implies that $\mathcal{K}$ contains the set of mixtures of exponentially distributed variables (but is bigger because uniformly distributed random variables are not mixtures of exponentially distributed variables).

Proposition 2.7 Let $\xi$ and $\xi^{\prime}$ be independent and exponentially identically (with mean 1) distributed random variables. Further, let $Z$ be a positive random variable independent of $\xi$ and $\xi^{\prime}$, and define $X=Z \xi$ and $X^{\prime}=Z \xi^{\prime}$. Then

$$
\left(X, X^{\prime}\right) \in \mathcal{K}
$$

Proof Letting $\varphi$ be a bounded and positive Borel function consider

$$
\begin{aligned}
\mathbf{E}\left(\varphi\left(X, X^{\prime}\right)\right) & =\mathbf{E}\left(\varphi\left(Z \xi, Z \xi^{\prime}\right)\right) \\
& =\int_{\mathbf{R}_{+}^{3}} \varphi(z u, z v) \mathrm{e}^{-(u+v)} d u d v F_{Z}(d z) \\
& =\int_{\mathbf{R}_{+}^{3}} \varphi(a, b) z^{-2} \mathrm{e}^{-(a+b) / z} d a d b F_{Z}(d z) \\
& =\int_{\mathbf{R}_{+}^{2}} \varphi(a, b) \rho(a+b) d a d b
\end{aligned}
$$


where

$$
\rho(s):=\mathbf{E}\left(Z^{-2} \exp (-s / Z)\right), \quad s>0 .
$$

Consequently, using Corollary 2.5, it is seen that $\left(X, X^{\prime}\right) \in \mathcal{K}$, as claimed.

Example 2.8 If $X$ and $X^{\prime}$ are two independent and exponentially (with identical means, $1 / \alpha$ say) distributed random variables, then $\left(X, X^{\prime}\right) \in \mathcal{K}$ by Proposition 2.7 (take $Z=1 / \alpha$ ). In this case, the variable $V$ in Definition 2.1 is $\Gamma(2, \alpha)$-distributed, i.e.,

$$
F_{V}(d v)=\alpha^{2} v \mathrm{e}^{-\alpha v} d v
$$

\section{Stopping a diffusion at randomised first range time}

In this section it is seen that the basic result in Vallois [32] Théorème 4 proved for Brownian motion when $\left(X, X^{\prime}\right)=(U, 1-U)$ is valid for all variables in $\mathcal{K}$ and all diffusions on $\mathbf{R}$.

Let $Z$ be a linear diffusion started at 0 and having the state space $\mathbf{R}$. Let $\left\{\mathcal{F}_{t}\right\}$ denote the natural filtration of $Z$. Define

$$
S_{t}:=\sup _{0 \leq s \leq t} Z_{s}, \quad I_{t}:=-\inf _{0 \leq s \leq t} Z_{s}, \quad R_{t}:=S_{t}+I_{t} .
$$

Further, introduce the right continuous inverse of $t \mapsto R_{t}$

$$
\theta(r):=\inf \left\{t: R_{t}>r\right\}, \quad r>0 .
$$

For a fixed $r>0$ we call $\theta(r)$ the first range time of the level $r$. Let

$$
H(a, b):=\inf \left\{t: Z_{t} \notin(-a, b)\right\}, \quad a, b>0,
$$

and

$$
H(c):= \begin{cases}\inf \left\{t: Z_{t}>c\right\}, & c>0 \\ \inf \left\{t: Z_{t}<c\right\}, & c<0 .\end{cases}
$$

Notice that $H(a, b)=\min (H(-a), H(b))$.

Proposition 3.1 Assume that $\left(X, X^{\prime}\right)$ is a positive 2-dimensional random variable independent of the diffusion $Z$. Then

$$
\left(X, X^{\prime}\right) \in \mathcal{K} \quad \Rightarrow \quad \theta(X) \stackrel{(\mathrm{d})}{=} H\left(X, X^{\prime}\right) .
$$


Proof Let for $s, t>0$

$$
F^{c}(s, t):=\mathbf{P}\left(X>s, X^{\prime}>t\right) .
$$

Because $\left(X, X^{\prime}\right)$ and $Z$ are assumed to be independent we have a.s.

$$
\mathbf{P}\left(\theta(X)>t \mid \mathcal{F}_{\infty}\right)=\mathbf{P}\left(R_{t}<X \mid \mathcal{F}_{\infty}\right)=F^{c}\left(R_{t}, 0\right)
$$

and

$$
\begin{aligned}
\mathbf{P}\left(H\left(X, X^{\prime}\right)>t \mid\right. & \left.\mathcal{F}_{\infty}\right)=\mathbf{P}\left(\min \left\{H(-X), H\left(X^{\prime}\right)\right\}>t \mid \mathcal{F}_{\infty}\right) \\
& =\mathbf{P}\left(H(-X)>t, H\left(X^{\prime}\right)>t \mid \mathcal{F}_{\infty}\right) \\
& =\mathbf{P}\left(I_{t}<X, S_{t}<X^{\prime} \mid \mathcal{F}_{\infty}\right) \\
& =F^{c}\left(I_{t}, S_{t}\right) .
\end{aligned}
$$

From Proposition 2.6 we know that

$$
F^{c}(s, t)=F^{c}(s+t, 0),
$$

and, consequently, as claimed

$$
\mathbf{P}(\theta(X)>t)=\mathbf{P}\left(H\left(X, X^{\prime}\right)>t\right) .
$$

Remark 3.2 From the proof of Proposition 3.1 it is seen that the identity (6) holds, in fact, conditionally given the whole trajectory of $Z$. For a converse of Proposition 3.1, notice that if we have a diffusion on $\mathbf{R}$ and a pair of random variables $\left(X, X^{\prime}\right)$ such that the conditional laws of $\theta(X)$ and $H\left(X, X^{\prime}\right)$ given $\mathcal{F}_{\infty}$ are the same then a.s.

$$
F^{c}\left(I_{t}, S_{t}\right)=F^{c}\left(R_{t}, 0\right)=F^{c}\left(I_{t}+S_{t}, 0\right)
$$

It can be proved that this implies that for all $x$ and $y$

$$
F^{c}(x, y)=F^{c}(x+y, 0)
$$

that is, $\left(X, X^{\prime}\right) \in \mathcal{K}$. 
Proposition 3.3 Assume that the 2-dimensional random variable $\left(X, X^{\prime}\right)$ belongs to $\mathcal{K}$, and is independent of the diffusion $Z$. Then

$$
\left\{Z_{s}: s \leq \theta(X)\right\} \stackrel{(\mathrm{d})}{=}\left\{Z_{s}: s \leq H\left(X, X^{\prime}\right)\right\} .
$$

Proof For simplicity, we prove the result in the case $\left(X, X^{\prime}\right)$ has a density. According to Corollary 2.5 there exists a function $\rho$ such that

$$
\mathbf{P}\left(X \in d a, X^{\prime} \in d b\right)=\rho(a+b) d a d b .
$$

For a non-negative integer $n$ let $0 \leq s_{1}<s_{2}<\ldots<s_{n}$ and $\varphi: \mathbf{R}^{n} \times \mathbf{R}_{+} \mapsto \mathbf{R}$ be a bounded and measurable function. Consider

$$
\begin{aligned}
\Delta_{+}:= & \int_{0}^{\infty} \int_{0}^{\infty} \varphi\left(Z_{s_{1}}, \ldots, Z_{s_{n}}, H(a, b)\right) \mathbf{1}_{\left\{s_{n}<H(a, b), Z_{H(a, b)}=b\right\}} \\
& \times \rho(a+b) d a d b \\
= & \int_{0}^{\infty} \int_{0}^{\infty} \varphi\left(Z_{s_{1}}, \ldots, Z_{s_{n}}, H(b)\right) 1_{\left\{s_{n}<H(b), I_{H(b)}<a\right\}} \rho(a+b) d a d b \\
= & \int_{0}^{\infty} \int_{0}^{\infty} \varphi\left(Z_{s_{1}}, \ldots, Z_{s_{n}}, t\right) \mathbf{1}_{\left\{s_{n}<t, I_{t}<a\right\}} \rho\left(a+S_{t}\right) d a d S_{t},
\end{aligned}
$$

where in the last step we have substituted $b=S_{t}$. Integrating with respect to $a$ gives

$$
\begin{aligned}
\Delta_{+}= & \int_{0}^{\infty} \varphi\left(Z_{s_{1}}, \ldots, Z_{s_{n}}, t\right) \mathbf{1}_{\left\{s_{n}<t\right\}}\left(\int_{I_{t}}^{\infty} \rho\left(a+S_{t}\right) d a\right) d S_{t} \\
& =\int_{0}^{\infty} \varphi\left(Z_{s_{1}}, \ldots, Z_{s_{n}}, t\right) \mathbf{1}_{\left\{s_{n}<t\right\}} \rho_{1}\left(I_{t}+S_{t}\right) d S_{t} \\
& =\int_{0}^{\infty} \varphi\left(Z_{s_{1}}, \ldots, Z_{s_{n}}, t\right) \mathbf{1}_{\left\{s_{n}<t\right\}} \rho_{1}\left(R_{t}\right) d S_{t},
\end{aligned}
$$

where

$$
\rho_{1}(u)=\int_{0}^{\infty} \rho(a+u) d a=\int_{u}^{\infty} \rho(s) d s
$$

is the density of $X$. Because $t \mapsto S_{t}$ can only increase when $Z_{t}>0$ and in this case also $d S_{t}=d R_{t}$ it follows that

$$
\Delta_{+}=\int_{0}^{\infty} \varphi\left(Z_{s_{1}}, \ldots, Z_{s_{n}}, t\right) \mathbf{1}_{\left\{s_{n}<t\right\}} \mathbf{1}_{\left\{Z_{t}>0\right\}} \rho_{1}\left(R_{t}\right) d R_{t}
$$


Substituting $r=R_{t}$ and recalling the notation $r \mapsto \theta(r)$ for the right continous inverse of $t \mapsto R_{t}$ we obtain

$$
\Delta_{+}=\int_{0}^{\infty} \varphi\left(Z_{s_{1}}, \ldots, Z_{s_{n}}, \theta(r)\right) \mathbf{1}_{\left\{s_{n}<\theta(r), Z_{\theta(r)}>0\right\}} \rho_{1}(r) d r .
$$

This completes the proof in the case $Z_{H(a, b)}=b$.

In the similar manner, for the case $Z_{H(a, b)}=-a$, considering

$$
\Delta_{-}:=\int_{0}^{\infty} \int_{0}^{\infty} \phi\left(Z_{s_{1}}, \ldots, Z_{s_{n}}, H(a, b)\right) 1_{\left\{s_{n}<H(a, b), Z_{H(a, b)}=-a\right\}} \rho(a+b) d a d b
$$

leads to

$$
\Delta_{-}=\int_{0}^{\infty} \phi\left(Z_{s_{1}}, \ldots, Z_{s_{n}}, \theta(r)\right) \mathbf{1}_{\left\{s_{n}<\theta(r), Z_{\theta(r)}<0\right\}} \rho_{1}(r) d r .
$$

The proof is now completed by adding the expressions of $\Delta_{+}$and $\Delta_{-}$.

\section{Ray-Knight theorem for Brownian motion stopped at the randomised range time}

\section{$4.1 \quad$ Results}

In this section we state the Ray-Knight theorem for the randomised range time, announced in the introduction. It is seen that the result in [5] No. VI.23 p. 118 follows from this as a corollary. The proof of the main result, Theorem 4.1, its generalization and the corollary are postponed to Section $4.2,4.3$, and 4.4, respectively. Throughout this section, it is assumed the underlying Brownian motion $W$ is started from 0 , and we let $\mathbf{P}_{0}$ and $\mathbf{E}_{0}$ denote the probability measure and the expectation, respectively, associated with $W$.

Let $\{L(t, y), t \geq 0, y \in \mathbf{R}\}$ denote the bicontinuous family of Brownian local times. We use the normalisation with respect to the Lebesgue measure, that is, we take

$$
L(t, y)=\lim _{\varepsilon \downarrow 0} \frac{1}{2 \varepsilon} \int_{0}^{t} \mathbf{1}_{(y-\varepsilon, y+\varepsilon)}\left(W_{s}\right) d s \quad \text { a.s. }
$$


The squared Bessel processes of dimension 2 and 4 play important roles in our study. We use notation $S Q B E S_{x}^{\delta}$, with $\delta=2$ and 4 , respectively, for these processes when started at $x$. When the initial point is of no importance we write simply $S Q B E S^{\delta}$. The generator of a $S Q B E S^{\delta}$ is

$$
\mathcal{G}^{\delta}=2 x \frac{d^{2}}{d x^{2}}+\delta \frac{d}{d x}, \quad x>0
$$

For $\delta=2$ or 4 the boundary point 0 is entrance-not-exit and $+\infty$ is natural (attracting) (see [5] A1.23 p. 135). In fact, the diffusions appearing in Theorem 4.1 are squared Bessel processes with downward drift. We use for these processes the notation $S Q B E S_{x}^{\delta, \alpha \downarrow}$, where $\alpha>0$ is the drift parameter and $\downarrow$ indicates that the process drifts towards 0 . Many properties of squared Bessel processes with drift can be studied using (ordinary) Bessel processes with drift. The latter ones have been investigated, e.g., in the papers by Watanabe [35], Pitman and Yor [21], and Vallois [30].

For the proof of Theorem 4.1 it is important to know that $S Q B E S^{\delta, \alpha \downarrow}$ can be constructed as an $h$-transform of $S Q B E S^{\delta}$. To explain this recall that the increasing and decreasing fundamental solutions of the ODE $\mathcal{G}^{\delta} f=\alpha f$ associated to $S Q B E S^{\delta}$ are (cf. [5] No. A1.23 p. 135)

$$
\psi_{\alpha}(x)=x^{-\nu / 2} I_{\nu}(\sqrt{2 \alpha x})
$$

and

$$
\varphi_{\alpha}(x)=x^{-\nu / 2} K_{\nu}(\sqrt{2 \alpha x}),
$$

respectively, where $\alpha>0, \nu=(\delta-2) / 2$, and $I_{\nu}$ and $K_{\nu}$ are modified Bessel functions of order $\nu$ (see Abramowitz and Stegun [1] p. 374). Consider now $S Q B E S^{\delta}$ killed at an exponential time $T$ having parameter $\alpha$; these are called killed $S Q B E S^{\delta}$ processes. As usual, it is assumed that $T$ is independent of $S Q B E S^{\delta}$. The function $\varphi_{\alpha}$ is excessive for killed $S Q B E S^{\delta}$. Now $S Q B E S^{\delta, \alpha \downarrow}$ is defined as the $h$-transform (cf. [5] II.31 p. 33) of killed $S Q B E S^{\delta}$ when we take $h=\varphi_{\alpha}$. Consequently, the generator of $S Q B E S^{\delta, \alpha \downarrow}$ is obtained as

$$
\begin{aligned}
\mathcal{G}^{\delta, \alpha \downarrow}(f) & =\frac{1}{\varphi_{\alpha}}(\mathcal{G}-\alpha)\left(\varphi_{\alpha} f\right) . \\
& =\mathcal{G}(f)(x)+4 x \frac{\varphi_{\alpha}^{\prime}(x)}{\varphi_{\alpha}(x)} \frac{d f}{d x}(x) \\
& =2 x \frac{d^{2} f}{d x^{2}}(x)+\left(2+2 \sqrt{2 \alpha x} \frac{K_{\nu}^{\prime}(\sqrt{2 \alpha x})}{K_{\nu}(\sqrt{2 \alpha x})}\right) \frac{d f}{d x}(x) .
\end{aligned}
$$


It can be verified (cf. [5] II.6 p.14) that 0 is an exit-not-entrance boundary point for $S Q B E S^{\delta, \alpha \downarrow}$. We remark also that $S Q B E S^{\delta, \alpha \downarrow}$ can interpreted as killed $S Q B E S^{\delta}$ conditioned to hit 0 (where the process is (really) killed).

Theorem 4.1 Let $\theta(r)$ be the first range time at level $r>0$ (cf. (5)) and $T$ an exponentially distributed random variable with parameter $\alpha>0$. Assume that $T$ is independent of the Brownian motion $W$. Let $\{L(\theta(T), y) ; y \in \mathbf{R}\}$ be the local time process of $W$ stopped at $\theta(T)$. Then for $l>0$

$$
\mathbf{P}_{0}(L(\theta(T), 0) \in d l)=2 \alpha \sqrt{2 \alpha l} K_{1}(\sqrt{2 \alpha l}) K_{0}(\sqrt{2 \alpha l}) d l .
$$

Conditionally on $W_{\theta(T)}>0$ and $L(\theta(T), 0)=l$

$$
\begin{gathered}
\{L(\theta(T),-y): y \geq 0\} \stackrel{(\mathrm{d})}{=}\left\{S Q B E S_{l}^{4, \alpha \downarrow}(y): y \geq 0\right\}, \\
\{L(\theta(T), y): y \geq 0\} \stackrel{(\mathrm{d})}{=}\left\{S Q B E S_{l}^{2, \alpha \downarrow}(y): y \geq 0\right\},
\end{gathered}
$$

where $S Q B E S_{l}^{4, \alpha \downarrow}$ and $S Q B E S_{l}^{2, \alpha \downarrow}$ are both started at $l$ but are otherwise independent.

Remark 4.2 By the spatial homogeneity of BM,

$$
\mathbf{P}_{0}\left(W_{\theta(T)}>0\right)=\mathbf{P}_{0}\left(W_{\theta(T)}<0\right)=\frac{1}{2}
$$

and, hence,

$$
\begin{aligned}
\mathbf{P}_{0}\left(L(\theta(T), 0) \in d l, W_{\theta(T)}>0\right) & =\frac{1}{2} \mathbf{P}_{0}(L(\theta(T), 0) \in d l) \\
& =\alpha \sqrt{2 \alpha l} K_{1}(\sqrt{2 \alpha l}) K_{0}(\sqrt{2 \alpha l}) d l .
\end{aligned}
$$

It is striking how the factor $2 \alpha$ appears in (10). This can be explained by the scaling property of BM. Moreover, the phenomenon allows us to generalize Theorem 4.1 (by randomising $\alpha$ ) as follows

Theorem 4.3 Let $T_{1}$ be an exponentially distributed random variable with parameter 1 , and $Z$ an arbitrary positive random variable. Assume that $T_{1}$, $Z$, and the Brownian motion $W$ are independent. Then for $l>0$

$$
\mathbf{P}_{0}\left(2 Z L\left(\theta\left(T_{1} / Z\right), 0\right) \in d l\right)=\sqrt{l} K_{1}(\sqrt{l}) K_{0}(\sqrt{l}) d l .
$$


Conditionally on $W_{\theta\left(T_{1} / Z\right)}>0$ and $Z L\left(\theta\left(T_{1} / Z\right), 0\right)=l$

$$
\begin{aligned}
& \left\{Z L\left(\theta\left(T_{1} / Z\right),-y / Z\right): y \geq 0\right\} \stackrel{(\mathrm{d})}{=}\left\{S Q B E S_{l}^{4,1 \downarrow}(y): y \geq 0\right\}, \\
& \left.\left\{Z L\left(\theta\left(T_{1} / Z\right), y / Z\right): y \geq 0\right\}\right\} \stackrel{(\mathrm{d})}{=}\left\{S Q B E S_{l}^{2,1 \downarrow}(y): y \geq 0\right\},
\end{aligned}
$$

where $S Q B E S_{l}^{4,1 \downarrow}$ and $S Q B E S_{l}^{2,1 \downarrow}$ are both started at $l$ but are otherwise independent.

Remark 4.4 It is possible to check by integration that the function on the right hand side of (11) (and also (10)) is a density. Indeed, we have

$$
\begin{aligned}
\int_{0}^{\infty} \sqrt{l} K_{1}(\sqrt{l}) K_{0}(\sqrt{l}) d l & =2 \int_{0}^{\infty} x^{2} K_{1}(x) K_{0}(x) d x \\
& =-2 \int_{0}^{\infty} x K_{1}(x) d\left(x K_{1}(x)\right) \\
& =1
\end{aligned}
$$

where we used the relations

$$
\frac{d}{d x}\left[x K_{1}(x)\right]=-x K_{0}(x) \quad \text { and } \quad K_{1}(x) \sim_{x \rightarrow 0} 1 / x
$$

(see Abramowitz and Stegun, formulae 9.6.28 p. 376 and 9.6.9 p. 375).

From Theorem 4.1 we obtain the following corollary, which is exactly the result in [5] No. VI.23 p.118, and, by Proposition 3.3, also the result in No. VI.14 p. 112 (when therein $\alpha=\beta$ ).

Corollary 4.5 Let the function $f$ be non-negative, continuous, and bounded. Assume also that $f(0)=0$ and define

$$
V(f, \theta(r)):=\int_{-\infty}^{+\infty} f(L(\theta(r), y)) d y .
$$

Then for $T$ as in Theorem 4.1 and $h>0$

$$
\begin{aligned}
\mathbf{E}_{0}\left(\exp (-V(f, \theta(T))) ; \sup _{y} L(\theta(T), y)<h\right) \\
=2 \alpha \int_{0}^{h} l \hat{G}_{\alpha}^{(2, f)}(0, l) \hat{G}_{\alpha}^{(4, f)}(0, l) d l
\end{aligned}
$$


where $\hat{G}_{\alpha}^{(2, f)}$ and $\hat{G}_{\alpha}^{(4, f)}$ are Green kernels associated to $S Q B E S^{2}$, denoted $Z^{(2)}$, and $S Q B E S^{4}$, denoted $Z^{(4)}$, respectively, when the processes are killed according to the additive functionals (for this, see [5] II.22 p.28)

$$
A_{t}^{(2)}:=\int_{0}^{t} f\left(Z_{s}^{(2)}\right) d s, \quad \text { and } \quad A_{t}^{(4)}:=\int_{0}^{t} f\left(Z_{s}^{(4)}\right) d s,
$$

respectively, and also if the processes exit the interval $(0, h)$.

This corollary gives the result in Theorem 4.1 in a form more suitable for computations. It is, e.g., possible to determine the distribution of $\sup _{y} L(\theta(T), y)$, see [5] 1.5.11.2, 1.5.14 p. 245.

Recall that the Green kernels can be expressed in terms of the fundamental solutions of the ordinary differential equation associated to the studied diffusion (see, e.g., [5] No. II.10 and 11 p. 18). For example, for the kernel $\hat{G}_{\alpha}^{(2, f)}$ we have the representation

$$
\hat{G}_{\alpha}^{(2, f)}(x, y)=w_{\alpha}^{-1} \varphi_{\alpha}^{(2, f)}(x) \psi_{\alpha}^{(2, f)}(y), \quad x \geq y,
$$

where $w_{\alpha}$ is the Wronskian. The functions $\varphi_{\alpha}^{(2, f)}$ and $\psi_{\alpha}^{(2, f)}$ are the unique (up to multiplicative constants) non-increasing and non-decreasing, respectively, solutions of the ODE

$$
2 x u^{\prime \prime}(x)+2 u^{\prime}(x)-(f(x)+\alpha) u(x)=0, \quad x>0,
$$

and $\varphi_{\alpha}^{(2, f)}$ must satisfy the boundary condition $u(h)=0$.

\subsection{Proof of Theorem 4.1}

We start with by recalling the Ray-Knight theorems from which Theorem 4.1 is deduced. The first one is for the local time process stopped at $H(b):=$ $\inf \{t: W(t)>b\}$ with $b>0$. The second one is for the local time stopped at $\theta(r)$.

Theorem 4.6 For $b>0$ and conditionally on $L(H(b), 0)=l$ and $I_{H(b)}=$ $-m$

$$
\begin{aligned}
& \{L(H(b),-y): 0 \leq y \leq-m\} \stackrel{(\mathrm{d})}{=}\left\{S Q B E S_{l \rightarrow 0}^{4,-m}(y): 0 \leq y \leq-m\right\}, \\
& \{L(H(b), y): 0 \leq y<b\} \stackrel{(\mathrm{d})}{=}\left\{S Q B E S_{l \rightarrow 0}^{2, b}(y): 0 \leq y \leq b\right\},
\end{aligned}
$$


where $\left\{S Q B E S_{l \rightarrow 0}^{\delta, a}(y): 0 \leq y \leq a\right\}$ denotes the $\delta$-dimensional squared Bessel process started at $l$ and conditioned to be at 0 at time $a$, also called $\delta$-dimensional squared Bessel bridge from $l$ to 0 with length $a$. The bridges $S Q B E S_{l \rightarrow 0}^{4,-m}$ and $S Q B E S_{l \rightarrow 0}^{2, b}$ are both started at $l$ but are otherwise independent.

Remark 4.7 Observe that, when compared with the result in Theorem 4.6 to [5] No. V.6 p. 85, we have here changed the $y$-direction in the process

$$
\{L(H(b),-y): 0 \leq y \leq-m\} .
$$

The change can be done by the time reversal property the diffusion bridges have (see Salminen [26]). In particular, it holds

$$
\left\{S Q B E S_{l \rightarrow 0}^{\delta, a}(y): 0 \leq y \leq a\right\} \stackrel{(\mathrm{d})}{=}\left\{S Q B E S_{0 \rightarrow l}^{\delta, a}(l-y): 0 \leq y \leq a\right\} .
$$

Remark 4.8 Squared Bessel bridges $S Q B E S_{l \rightarrow 0}^{\delta, a}$ can be defined, or constructed, as Doob's $h$-transforms (in space-time) of $S Q B E S_{l}^{\delta}$ taking

$$
h(t, x)=p(a-t ; x, 0), \quad 0 \leq t<a
$$

where $p$ is the transition density of $S Q B E S^{\delta}$. This construction corresponds the intuition of conditioning $S Q B E S_{l}^{\delta}$ to be at 0 at time $a$. Notice that because 0 is an entrance boundary point for $S Q B E S^{\delta}$ we have $S Q B E S_{l \rightarrow 0}^{\delta, a}(y)>$ 0 for all $0 \leq y<a$. For general theory of $h$-transforms, see Sharpe [27] and for diffusions Salminen [25].

The Ray-Knight theorem for the local time process stopped at the first range time $\theta(r):=\inf \left\{t: S_{t}+I_{t}>r\right\}$ is obtained from Theorem 4.6 by observing that, under $\mathbf{P}_{0}$,

$$
W_{\theta(r)}=z, \quad r>z>0 \quad \Leftrightarrow \quad I_{H(z)}=r-z .
$$

Notice also that if $W_{\theta(r)}=z$ then $\theta(r)=H(z)$. Now we have (see Vallois [31] for generalizations)

Theorem 4.9 Conditionally on $W_{\theta(r)}=z, r>z>0$, and $L(\theta(r), 0)=l$

$$
\begin{gathered}
\{L(\theta(r),-y): 0 \leq y \leq r-z\} \stackrel{(\mathrm{d})}{=}\left\{S Q B E S_{l \rightarrow 0}^{4, r-z}(y): 0 \leq y \leq r-z\right\}, \\
\{L(\theta(r), y): 0 \leq y<z\} \stackrel{(\mathrm{d})}{=}\left\{S Q B E S_{l \rightarrow 0}^{2, z}(y): 0 \leq y \leq z\right\},
\end{gathered}
$$

where $S Q B E S_{l \rightarrow 0}^{4, r-z}$ and $S Q B E S_{l \rightarrow 0}^{2, z}$ are both started at $l$ but are otherwise independent. 
To be able to apply Theorem 4.9 we need the distributions of $W_{\theta(r)}$ and $L(\theta(r), 0)$. These are given in the next proposition.

\section{Proposition 4.10}

$$
\begin{gathered}
\mathbf{P}_{0}\left(W_{\theta(r)} \in d z\right)=\frac{|z|}{r^{2}} d z, \quad|z| \leq r \\
\mathbf{P}_{0}\left(L(\theta(r), 0) \in d l \mid W_{\theta(r)}=z\right)=\eta^{2} l \mathrm{e}^{-\eta l} d l, \quad \eta:=r /(2(r-|z|)|z|), \\
\mathbf{P}_{0}(L(\theta(r), 0) \in d l)=\frac{l}{r^{2}} \mathrm{e}^{-l / r}\left(K_{0}(l / r)+K_{1}(l / r)\right) d l
\end{gathered}
$$

Proof For (12) and (13), see, e.g., [5] VI.22 p. 117 and V.7 p. 86, respectively. To prove (14) use (12) and (13) to obtain

$$
\begin{aligned}
\mathbf{P}_{0}(L(\theta(r), 0) \in d l) / d l & =\int_{|z| \leq r} \frac{|z| \eta^{2} l}{r^{2}} \exp (-\eta l) d z \\
& =\frac{l}{2} \int_{0}^{r} \frac{1}{(r-z)^{2} z} \exp \left(-\frac{l r}{2(r-z) z}\right) d z \\
& =\frac{l}{2 r^{2}} \mathrm{e}^{-\frac{l}{r}} \int_{0}^{+\infty}\left(\frac{1}{u}+\frac{1}{u^{2}}\right) \exp \left(-\frac{l}{2 r}\left(u+\frac{1}{u}\right)\right) d u
\end{aligned}
$$

where in the last step we have substituted $z=r /(u+1)$. The formula (14) follows from the integral representation (see Watson [36] p. 183 and also (15) in Proposition 4.11)

$$
K_{\nu}(\sqrt{l})=\frac{l^{\nu / 2}}{2} \int_{0}^{\infty} t^{-\nu-1} \exp \left(-\frac{1}{2}\left(t+\frac{l}{t}\right)\right) d t
$$

To proceed, we recall the following result (see Barndorff-Nielsen, Blaesild and Halgreen [2], Salminen [25], and Vallois [30]), which can be proved, e.g., using last exit times.

Proposition 4.11 The first hitting time

$$
H_{l}^{\delta, \alpha \downarrow}(0):=\inf \left\{t: S Q B E S_{l}^{\delta, \alpha \downarrow}(t)=0\right\}
$$


is distributed according to the generalized inverse Gaussian law, that is,

$$
\begin{aligned}
\mathbf{P}\left(H_{l}^{\delta, \alpha \downarrow}(0)\right. & \in d t) \\
& =\left(\frac{l}{2 \alpha}\right)^{\nu / 2} \frac{1}{2 K_{\nu}(\sqrt{2 \alpha l})} t^{-\nu-1} \exp \left(-\alpha t-\frac{l}{2 t}\right) d t \\
& =: n_{0}(t, l) d t
\end{aligned}
$$

where $\delta=2 \nu+2$.

Next proposition is the key to Theorem 4.1. Let for $\delta \geq 2 S Q B E S_{l \rightarrow 0}^{\delta, \alpha \downarrow, a}$ denote the $S Q B E S^{\delta, \alpha \downarrow}$ started at $l$ and conditioned to hit 0 at time $a$. Also $S Q B E S_{l \rightarrow 0}^{\delta, \alpha \downarrow, a}$ can be constructed as Doob's $h$-transform, but here we cannot use the transition density of $S Q B E S^{\delta, \alpha \downarrow}$ (as is done for $S Q B E S^{\delta}$ bridges, cf. Remark 4.8) because this density has value 0 when one argument has the value 0 . Instead we take

$$
h(t, x):=n_{0}(a-t, x), \quad 0 \leq t<a,
$$

where $n_{0}(\cdot, l)$ is the density of the first hitting time $H_{l}^{\delta, \alpha \downarrow}(0)$, as given in Proposition 4.11 .

Proposition 4.12 For $\delta \geq 2$ and $\alpha>0$ the bridges $S Q B E S_{l \rightarrow 0}^{\delta, a}$ and $S Q B E S_{l \rightarrow 0}^{\delta, \alpha \downarrow, a}$ are identical in law.

Proof We prove the claim by checking that the Radon-Nikodym derivatives of measures associated to $S Q B E S_{l \rightarrow 0}^{\delta, a}$ and $S Q B E S_{l \rightarrow 0}^{\delta, \alpha \downarrow, a}$ with respect to the measure of $S Q B E S_{l}^{\delta}$ are the same. For this, we work in the usual canonical setting of continuous functions $t \mapsto \omega(t), t \geq 0$, and let $\mathcal{F}_{t}$ be the smallest $\sigma$-algebra generated by $\omega(s), s \leq t$. Letting $F_{t}$ be an $\mathcal{F}_{t^{-}}$ measurable and bounded random variable and using the $h$-transform description of $S Q B E S_{l \rightarrow 0}^{\delta, a}$ and $S Q B E S_{l \rightarrow 0}^{\delta, \alpha \downarrow, a}$ we obtain for $t<a$

$$
\mathbf{E}_{l \rightarrow 0}^{\delta, a}\left(F_{t}\right)=\mathbf{E}_{l}^{\delta}\left(\frac{p(a-t ; \omega(t), 0)}{p(a ; l, 0)} F_{t} ; t<H_{0}\right)
$$

and, by using the Cameron-Martin transform

$$
\begin{aligned}
\mathbf{E}_{l \rightarrow 0}^{\delta, \alpha \downarrow, a}\left(F_{t}\right) & =\mathbf{E}_{l}^{\delta, \alpha \downarrow}\left(\frac{n_{0}(a-t, \omega(t))}{n_{0}(a, l)} F_{t} ; t<H_{0}\right) \\
& =\mathbf{E}_{l}^{\delta}\left(\frac{\mathrm{e}^{\alpha(a-t)} n_{0}(a-t, \omega(t)) \varphi_{\alpha}(\omega(t))}{\mathrm{e}^{\alpha a} n_{0}(a, l) \varphi_{\alpha}(l)} F_{t} ; t<H_{0}\right),
\end{aligned}
$$


where $p$ is the transition density of $S Q B E S^{\delta}$ and $n_{0}$ is the density of the first hitting time

$$
H_{0}:=\inf \{t: \omega(t)=0\}
$$

of $S Q B E S^{\delta, \alpha \downarrow}$, as given in Proposition 4.10. Because (see, e.g., [5] p. 136) for some constant $C$

$$
p(a ; l, 0)=p(a ; 0, l)=C a^{-\nu-1} \exp \left(-\frac{l}{2 a}\right)
$$

straightforward computations using (8) and (15) show that the derivatives in (16) and (17) are equal.

Remark 4.13 We have stated in Proposition 4.12 a particular case of a general result which informally says that if two diffusions are $h$-transforms of each other then the bridges constructed from these processes are identical in law. This fact, in a slightly weaker form because the end point of our bridges is not in the state space, is observed in Pitman and Yor [21] Remark (3.7) p. 306. We also refer to Pitman and Yor [22]. The connection between Doob's transforms and conditioning of diffusions, also diffusion bridges, appears (perhaps for the first time) in McKean [20] p. 236 where these concepts are applied in excursion theory. This approach is further developed in Williams [37]. We also refer to Salminen [24] for 3-dimensional Bessel bridges and to Fitzsimmons [11] for a more general framework.

We can now put the pieces together and complete the proof of Theorem 4.1. Let $\Phi_{+}$and $\Phi_{-}$be arbitrary measurable functionals of continuous functions on $\mathbf{R}_{+}$. We wish to compute

$$
\begin{aligned}
\Delta_{+}:=\mathbf{E}_{0}\left(\Phi_{+}(\{\right. & L(\theta(T), y) ; y \geq 0\}) \\
& \left.\left.\times \Phi_{-}(\{L(\theta(T),-y) ; y \geq 0\}) ; W_{\theta(T)}>0\right)\right) .
\end{aligned}
$$

To make the notation shorter, we omit the arguments for $\Phi_{ \pm}$and write simply

$$
\Phi_{+}=\Phi_{+}(\{L(\theta(T), y) ; y \geq 0\}), \quad \Phi_{-}=\Phi_{-}(\{L(\theta(T),-y) ; y \geq 0\}) .
$$

Clearly,

$$
\begin{aligned}
& \Delta_{+}=\mathbf{E}_{0}\left(\Phi_{+} \Phi_{-} ; W_{\theta(T)}>0\right) \\
& =\int_{\mathbf{R}^{3}} \alpha \mathrm{e}^{-\alpha r} \mathbf{E}_{0}\left(\Phi_{+} \Phi_{-} \mid L(\theta(r), 0)=l, W_{\theta(r)}=z\right) \mathbf{1}_{\{r>0,0<l, 0<z<r\}} \\
& \times \mathbf{P}_{0}\left(L(\theta(r), 0) \in d l \mid W_{\theta(r)}=z\right) \mathbf{P}_{0}\left(W_{\theta(r)} \in d z\right) d r .
\end{aligned}
$$


From Theorem 4.9 we obtain

$$
\mathbf{E}_{0}\left(\Phi_{+} \Phi_{-} \mid L(\theta(r), 0)=l, W_{\theta(r)}=z\right)=F_{+}^{(2)}(z, l) F_{-}^{(4)}(r-z, l),
$$

where

$$
F_{+}^{(2)}(z, l):=\mathbf{E}\left(\Phi_{+}\left(\left\{S Q B E S_{l \rightarrow 0}^{2, z}(y): 0 \leq y \leq z\right\}\right)\right)
$$

and

$$
F_{-}^{(4)}(s, l):=\mathbf{E}\left(\Phi_{-}\left(\left\{S Q B E S_{l \rightarrow 0}^{4, s}(y): 0 \leq y \leq s\right\}\right)\right) .
$$

Changing variables and using the explicit form of the joint distribution of $L(\theta(r), 0)$ and $W_{\theta(r)}$ (see Proposition 4.10) yields

$$
\begin{aligned}
& \Delta_{+}=\alpha \int_{\mathbf{R}_{+}^{3}} F_{+}^{(2)}(z, l) F_{-}^{(4)}(s, l) e^{-\alpha(s+z)} \frac{l}{4 s^{2} z} \exp \left(-\frac{s+z}{2 s z} l\right) d l d s d z \\
&=\alpha \int_{0}^{\infty}\left(\int_{0}^{\infty} F_{+}^{(2)}(z, l) \frac{1}{2 z} \exp \left(-\alpha z-\frac{l}{2 z}\right) d z\right) \\
& \quad \times\left(\int_{0}^{\infty} F_{-}^{(4)}(s, l) \frac{1}{2 s^{2}} \exp \left(-\alpha s-\frac{l}{2 s}\right) d s\right) l d l
\end{aligned}
$$

To proceed, consider the first integral inside the integral in the expression for $\Delta_{+}$given in (18) above. We obtain from (15)

$$
\begin{aligned}
\int_{0}^{\infty} & F_{+}^{(2)}(z, l) \frac{1}{2 z} \exp \left(-\alpha z-\frac{l}{2 z}\right) d z \\
& =K_{0}(\sqrt{2 \alpha l}) \int_{0}^{\infty} F_{+}^{(2)}(z, l) \mathbf{P}\left(H_{l}^{2, \alpha \downarrow}(0) \in d z\right) .
\end{aligned}
$$

By Proposition 4.12,

$$
F_{+}^{(2)}(z, l)=\mathbf{E}\left(\Phi_{+}\left(\left\{S Q B E S_{l \rightarrow 0}^{2, \alpha \downarrow, z}(y): 0 \leq y \leq z\right\}\right)\right) .
$$

Consequently, because $S Q B E S^{2, \alpha \downarrow}$ hits 0 with probability 1 and because 0 is an exit boundary we obtain by a standard monotone class argument

$$
\begin{aligned}
\int_{0}^{\infty} & F_{+}^{(2)}(z, l) \mathbf{P}\left(H_{l}^{2, \alpha \downarrow}(0) \in d z\right) \\
= & \int_{0}^{\infty} \mathbf{E}\left(\Phi_{+}\left(\left\{S Q B E S_{l \rightarrow 0}^{2, \alpha \downarrow, z}(y): 0 \leq y \leq z\right\}\right)\right) \mathbf{P}\left(H_{l}^{2, \alpha \downarrow}(0) \in d z\right) \\
= & \mathbf{E}\left(\Phi_{+}\left(\left\{S Q B E S_{l}^{2, \alpha \downarrow}(y): y \geq 0\right\}\right)\right)
\end{aligned}
$$


giving

$$
\begin{aligned}
& \int_{0}^{\infty} F_{+}^{(2)}(z, l) \frac{1}{2 z} \exp \left(-\alpha z-\frac{l}{2 z}\right) d z \\
& \quad=K_{0}(\sqrt{2 \alpha l}) \mathbf{E}\left(\Phi_{+}\left(\left\{S Q B E S_{l}^{2, \alpha \downarrow}(y): y \geq 0\right\}\right)\right) .
\end{aligned}
$$

We compute the second integral in (18) similarly. By Proposition 4.12

$$
F_{-}^{(4)}(s, l)=\mathbf{E}\left(\Phi_{-}\left(\left\{\operatorname{SQBEE} S_{l \rightarrow 0}^{4, \alpha \downarrow, s}(y): 0 \leq y \leq s\right\}\right)\right)
$$

and so, using again (15),

$$
\begin{aligned}
\int_{0}^{\infty} F_{-}^{(4)}(s, & l) \frac{1}{2 s^{2}} \exp \left(-\alpha s-\frac{l}{2 s}\right) d s \\
= & \left(\frac{2 \alpha}{l}\right)^{1 / 2} K_{1}(\sqrt{2 \alpha l}) \int_{0}^{\infty} F_{-}^{(4)}(s, l) \mathbf{P}\left(H_{l}^{4, \alpha \downarrow}(0) \in d s\right) \\
& =\left(\frac{2 \alpha}{l}\right)^{1 / 2} K_{1}(\sqrt{2 \alpha l}) \mathbf{E}\left(\Phi_{-}\left(\left\{S Q B E S_{l}^{4, \alpha \downarrow}(y): y \geq 0\right\}\right)\right) .
\end{aligned}
$$

Choosing $\Phi_{-} \equiv 1$ and $\Phi_{+}\left(\left\{X_{y}: y \geq 0\right\}\right)=\phi\left(X_{0}\right)$ where $\phi$ is any measurable function and $y \mapsto X_{y}$ is a continuous function, we obtain on one hand, by (18)

$$
\begin{aligned}
\Delta_{+}=\alpha \int_{0}^{\infty} & K_{0}(\sqrt{2 \alpha l}) \mathbf{E}\left(\Phi_{+}\left(\left\{S Q B E S_{l}^{2, \alpha \downarrow}(y): y \geq 0\right\}\right)\right) \\
& \times\left(\frac{2 \alpha}{l}\right)^{1 / 2} K_{1}(\sqrt{2 \alpha l}) \mathbf{E}\left(\Phi_{-}\left(\left\{S Q B E S_{l}^{4, \alpha \downarrow}(y): y \geq 0\right\}\right)\right) l d l \\
= & \sqrt{2} \alpha^{3 / 2} \int_{0}^{\infty} K_{1}(\sqrt{2 \alpha l}) K_{0}(\sqrt{2 \alpha l}) \sqrt{l} \phi(l) d l
\end{aligned}
$$

and, on the other hand,

$$
\begin{aligned}
\Delta_{+} & =\mathbf{E}_{0}\left(\Phi_{+} \Phi_{-} ; W_{\theta(T)}>0\right) \\
& =\mathbf{E}_{0}\left(\phi(L(\theta(T), 0)) ; W_{\theta(T)}>0\right) \\
& =\int_{0}^{\infty} \phi(l) \mathbf{P}_{0}\left(L(\theta(T), 0) \in d l, W_{\theta(T)}>0\right)
\end{aligned}
$$


from which we derive

$$
\mathbf{P}_{0}\left(L(\theta(T), 0) \in d l, W_{\theta(T)}>0\right)=\alpha \sqrt{2 \alpha l} K_{1}(\sqrt{2 \alpha l}) K_{0}(\sqrt{2 \alpha l}) d l .
$$

By symmetry, the same results hold when replacing $W_{\theta(T)}>0$ by $W_{\theta(T)}<0$ which proves (10). Now, choosing for $\Phi_{+}$a general function of the form $\phi \times \Phi_{+}$where $\phi$ is a function of $X_{0}$ as above and choosing a general function $\Phi$ - we first get

$$
\begin{aligned}
& \Delta_{+}=\int_{0}^{\infty} \phi(l) \mathbf{E}\left(\Phi_{+}\left(\left\{S Q B E S_{l}^{2, \alpha \downarrow}(y): y \geq 0\right\}\right)\right) \\
& \times \mathbf{E}\left(\Phi_{-}\left(\left\{S Q B E S_{l}^{4, \alpha \downarrow}(y): y \geq 0\right\}\right)\right) \\
& \times \mathbf{P}_{0}\left(L(\theta(T), 0) \in d l, W_{\theta(T)}>0\right)
\end{aligned}
$$

and second

$$
\begin{aligned}
& \Delta_{+}= \mathbf{E}_{0}\left(\Phi_{+} \Phi_{-}, W_{\theta(T)}>0\right) \\
&=\int_{0}^{\infty} \phi(l) \mathbf{E}_{0}\left(\Phi_{+} \Phi_{-} \mid W_{\theta(T)}>0, L(\theta(T), 0)=l\right) \\
& \quad \times \mathbf{P}_{0}\left(L(\theta(T), 0) \in d l, W_{\theta(T)}>0\right)
\end{aligned}
$$

which proves by identification

$$
\begin{aligned}
\mathbf{E}_{0}\left(\Phi_{+} \Phi_{-} \mid W_{\theta(T)}>0,\right. & L(\theta(T), 0)=l) \\
=\mathbf{E}\left(\Phi_{+}\left(\left\{S Q B E S_{l}^{2, \alpha \downarrow}(y): y \geq 0\right\}\right)\right) & \\
\quad & \mathbf{E}\left(\Phi_{-}\left(\left\{S Q B E S_{l}^{4, \alpha \downarrow}(y): y \geq 0\right\}\right)\right) .
\end{aligned}
$$

This completes the proof of Theorem 4.1.

Remark 4.14 As pointed out by the referee, it is possible to compute the distribution of $L(\theta(T), 0)$ directly from (14) in Proposition 4.10. Indeed,

$$
\begin{aligned}
\mathbf{P}_{0}(L(\theta(T), 0) \in d l) / d l & =\int_{0}^{+\infty} d r \alpha \mathrm{e}^{-\alpha r} \mathbf{P}_{0}(L(\theta(r), 0) \in d l) / d l \\
& =\int_{0}^{+\infty} \frac{\alpha l}{r^{2}} \mathrm{e}^{-\alpha r-\frac{l}{r}}\left(K_{0}(l / r)+K_{1}(l / r)\right) d r \\
& =\alpha \int_{0}^{+\infty} \mathrm{e}^{-r-\frac{\alpha l}{r}}\left(K_{0}(r)+K_{1}(r)\right) d r
\end{aligned}
$$


To proceed we need the formula no. 6.653 (2) in Gradshteyn and Ryzhik [13] p. 725 due to MacDonald (see Watson [36] p. 439):

$$
\int_{0}^{+\infty} \exp \left(-\frac{1}{2}\left(x+\frac{a^{2}+b^{2}}{x}\right)\right) K_{\nu}\left(\frac{a b}{x}\right) \frac{d x}{x}=2 K_{\nu}(a) K_{\nu}(b) .
$$

Taking here $a=b$ and changing variables give

$$
\int_{0}^{+\infty} \mathrm{e}^{-x-\frac{a^{2}}{2 x}} K_{\nu}(x) \frac{d x}{x}=2 K_{\nu}(a)^{2} .
$$

Let $\gamma=\sqrt{2 \alpha l}$ and consider

$$
\begin{aligned}
\int_{0}^{+\infty} \mathrm{e}^{-r-\frac{\gamma^{2}}{2 r}} r K_{0}(r) d r & =-\int_{0}^{+\infty} \mathrm{e}^{-r-\frac{\gamma^{2}}{2 r}} \frac{d}{d r}\left(r K_{1}(r)\right) d r \\
& =\int_{0}^{+\infty}\left(-1+\frac{\gamma^{2}}{2 r^{2}}\right) \mathrm{e}^{-r-\frac{\gamma^{2}}{2 r}} r K_{1}(r) d r
\end{aligned}
$$

where we have used (cf. Remark 4.4)

$$
\frac{d}{d x}\left[x K_{1}(x)\right]=-x K_{0}(x) .
$$

Consequently, by (19),

$$
\begin{aligned}
\int_{0}^{+\infty} \mathrm{e}^{-r-\frac{\gamma^{2}}{2 r}} r\left(K_{0}(r)+K_{1}(r)\right) d r & =\frac{\gamma^{2}}{2} \int_{0}^{+\infty} \mathrm{e}^{-r-\frac{\gamma^{2}}{2 r}} K_{1}(r) \frac{d r}{r} \\
& =\gamma^{2} K_{1}(\gamma)^{2} .
\end{aligned}
$$

Differentiating with respect to $\gamma$ gives

$$
\begin{aligned}
\int_{0}^{+\infty} \mathrm{e}^{-r-\frac{\gamma^{2}}{2 r}}\left(K_{0}(r)+K_{1}(r)\right) d r & =\frac{1}{-\gamma} 2 \gamma K_{1}(\gamma) \frac{d}{d \gamma}\left(\gamma K_{1}(\gamma)\right) \\
& =-2 K_{1}(\gamma) \frac{d}{d \gamma}\left(\gamma K_{1}(\gamma)\right) \\
& =2 \gamma K_{0}(\gamma) K_{1}(\gamma)
\end{aligned}
$$

proving the claim. 


\subsection{Proof of Theorem 4.3}

For $\alpha>0$ let $T_{\alpha}:=T_{1} / \alpha$. Then $T_{\alpha}$ is exponentially distributed with parameter $\alpha$, and we obtain from (10) by changing variables

$$
\mathbf{P}_{0}\left(2 \alpha L\left(\theta\left(T_{\alpha}\right), 0\right) \in d l\right)=\sqrt{l} K_{1}(\sqrt{l}) K_{0}(\sqrt{l}) d l .
$$

This gives immediately (11) because $Z$ is assumed to be independent of $T_{1}$ and $W$.

The remaining claim of Theorem 4.3 follows from Theorem 4.1 if we show that

$$
\left\{\alpha L\left(\theta\left(T_{\alpha}\right), y / \alpha\right): y \in \mathbf{R}\right\} \stackrel{(\mathrm{d})}{=}\left\{L\left(\theta\left(T_{1}\right), y\right): y \in \mathbf{R}\right\} .
$$

To prove this, recall that by the scaling property of $\mathrm{BM}$, under $\mathbf{P}_{0}$,

$$
\left\{\left(W_{t}, S_{t}, I_{t}\right): t \geq 0\right\} \stackrel{(\mathrm{d})}{=}\left\{\alpha\left(W_{t / \alpha^{2}}, S_{t / \alpha^{2}}, I_{t / \alpha^{2}}\right): t \geq 0\right\}
$$

It follows that

$$
\begin{aligned}
\theta(r):=\inf & \left\{t: S_{t}+I_{t}>r\right\} \\
& \stackrel{(\mathrm{d})}{=} \inf \left\{t: S_{t / \alpha^{2}}+I_{t / \alpha^{2}}>r / \alpha\right\} \\
& \stackrel{(\mathrm{d})}{=} \alpha^{2} \inf \left\{t: S_{t}+I_{t}>r / \alpha\right\} \\
& =\alpha^{2} \theta(r / \alpha) .
\end{aligned}
$$

Let $f$ be a bounded and non-negative function and consider

$$
\begin{aligned}
\int f(x) L(\theta(r), x) d x & =\int_{0}^{\theta(r)} f\left(W_{s}\right) d s \\
& \stackrel{(\mathrm{d})}{=} \int_{0}^{\alpha^{2} \theta(r / \alpha)} f\left(\alpha W_{s / \alpha^{2}}\right) d s \\
& =\alpha^{2} \int_{0}^{\theta(r / \alpha)} f\left(\alpha W_{s}\right) d s \\
& =\alpha^{2} \int f(\alpha x) L(\theta(r / \alpha), x) d x \\
& =\alpha \int f(y) L(\theta(r / \alpha), y / \alpha) d y
\end{aligned}
$$

and this proves (21). 
Remark 4.15 It is easily deduced from the explicit form of the transition density of $S Q B E S^{\delta}$ given, e.g., in [5] p. 135 that the following scaling property holds

$$
\left\{S Q B E S_{x}^{\delta}(t): t \geq 0\right\} \stackrel{(\mathrm{d})}{=}\left\{\frac{1}{c} S Q B E S_{c x}^{\delta}(c t): t \geq 0\right\}
$$

Using this and the $h$-transform description of $S Q B E S^{\delta, \alpha \downarrow}$ it can be proved that $S Q B E S^{\delta, \alpha \downarrow}$ (and also $S Q B E S^{\delta, \alpha \uparrow}$ ) satisfies

$$
\left\{S Q B E S_{x}^{\delta, \alpha \downarrow}(t): t \geq 0\right\} \stackrel{(\mathrm{d})}{=}\left\{\frac{1}{c} S Q B E S_{c x}^{\delta, \alpha / c \downarrow}(c t): t \geq 0\right\} .
$$

\subsection{Proof of Corollary 4.5}

For an (arbitrary) process $X=\left\{X_{y}: y \geq 0\right\}$ define

$$
\Phi(X):=\exp \left(-\int_{0}^{+\infty} f\left(X_{y}\right) d y\right) \mathbf{1}_{\left\{\sup _{y \geq 0} X_{y}<h\right\}} .
$$

By Theorem 4.1,

$$
\begin{aligned}
\mathbf{E}_{0}\left(\exp (-V(f, \theta(T))) ; \sup _{y} L(\theta(T), y)<h\right) \\
=\int_{0}^{h} \Psi_{+}(l) \Psi_{-}(l) 2 \alpha \sqrt{2 \alpha l} K_{1}(\sqrt{2 \alpha l}) K_{0}(\sqrt{2 \alpha l}) d l
\end{aligned}
$$

where

$$
\Psi_{+}(l):=\mathbf{E}\left(\Phi\left(S Q B E S_{l}^{2, \alpha \downarrow}\right)\right)
$$

and

$$
\Psi_{-}(l):=\mathbf{E}\left(\Phi\left(S Q B E S_{l}^{4, \alpha \downarrow}\right)\right) .
$$

As in the proof of Theorem 4.1, using Proposition 4.11 and 4.12, we obtain

$$
\begin{aligned}
\Psi_{+}(l)=\int_{0}^{+\infty} \mathbf{E}\left(\Phi\left(S Q B E S_{l \rightarrow 0}^{2, \alpha \downarrow, z}\right)\right) \mathbf{P}\left(H_{l}^{2, \alpha \downarrow}(0) \in d z\right) \\
=\int_{0}^{+\infty} \mathbf{E}\left(\Phi\left(S Q B E S_{l \rightarrow 0}^{2, z}\right)\right) \\
\quad \times \frac{1}{2 K_{0}(\sqrt{2 \alpha l})} \frac{1}{z} \exp \left(-\alpha z-\frac{l}{2 z}\right) d z
\end{aligned}
$$


and by the same way

$$
\begin{aligned}
\Psi_{-}(l)=\int_{0}^{+\infty} \mathbf{E} & \left(\Phi\left(S Q B E S_{l \rightarrow 0}^{4, y}\right)\right) \\
& \times \frac{l}{2 \sqrt{2 \alpha l} K_{1}(\sqrt{2 \alpha l})} \frac{1}{y^{2}} \exp \left(-\alpha y-\frac{l}{2 y}\right) d y .
\end{aligned}
$$

Next let $Z^{(2)}$ and $Z^{(4)}$ denote $S Q B E S^{2}$ and $S Q B E S^{4}$, respectively, and $p^{(2)}$ and $p^{(4)}$ their transition densities, respectively, normalized via

$$
\mathbf{E}\left(Z_{z}^{(2)} \in d u \mid Z_{0}^{(2)}=l\right)=p^{(2)}(z ; l, u) m^{(2)}(d u)
$$

and

$$
\mathbf{E}\left(Z_{z}^{(4)} \in d u \mid Z_{0}^{(4)}=l\right)=p^{(4)}(z ; l, u) m^{(4)}(d u)
$$

where $m^{(2)}(d u)=d u$ and $m^{(4)}(d u)=\frac{u}{2} d u$ are the speed measures of $S Q B E S^{2}$ and $S Q B E S^{4}$, respectively. In particular,

$$
p^{(2)}(z ; 0, l)=p^{(2)}(z ; l, 0)=\frac{1}{2 z} \exp \left(-\frac{l}{2 z}\right)
$$

and

$$
p^{(4)}(z ; 0, l)=p^{(4)}(z ; l, 0)=\frac{1}{2 z^{2}} \exp \left(-\frac{l}{2 z}\right) .
$$

Also define the transition densities $\hat{p}^{(2)}$ and $\hat{p}^{(4)}$ (which depend on $h$ and $f$ ) via

$$
\begin{gathered}
\mathbf{E}\left(\exp \left(-\int_{0}^{z} f\left(Z_{t}^{(2)}\right) d t\right) ; \sup _{0 \leq t \leq z} Z_{t}^{(2)}<h, Z_{z}^{(2)} \in d u \mid Z_{0}^{(2)}=l\right) \\
=\hat{p}^{(2)}(z ; l, u) m^{(2)}(d u)
\end{gathered}
$$

and

$$
\begin{gathered}
\mathbf{E}\left(\exp \left(-\int_{0}^{z} f\left(Z_{t}^{(4)}\right) d t\right) ; \sup _{0 \leq t \leq z} Z_{t}^{(4)}<h, Z_{z}^{(4)} \in d u \mid Z_{0}^{(4)}=l\right) \\
=\hat{p}^{(4)}(z ; l, u) m^{(4)}(d u) .
\end{gathered}
$$

Because $f$ is assumed to be bounded, the boundary point 0 is entrance-notexit for these killed $S Q B E S$ processes. Therefore, we have for all $l>0$

$$
\hat{p}^{(2)}(z ; l, 0)>0 \quad \text { and } \quad \hat{p}^{(4)}(z ; l, 0)>0 .
$$


Relations (24), (28), and (26) yield

$\mathbf{E}\left(\Phi\left(S Q B E S_{l \rightarrow 0}^{2, z}\right)\right)$

$$
\begin{aligned}
& =\mathbf{E}\left(\exp \left(-\int_{0}^{z} f\left(Z_{t}^{(2)}\right) d t\right) ; \sup _{0 \leq t \leq z} Z_{t}^{(2)}<h \mid Z_{0}^{(2)}=l, Z_{z}^{(2)}=0\right) \\
& =\frac{\hat{p}^{(2)}(z ; l, 0)}{p^{(2)}(z ; l, 0)} \\
& =2 z \exp \left(\frac{l}{2 z}\right) \hat{p}^{(2)}(z ; l, 0) .
\end{aligned}
$$

Similarly, by (25), (29), and (27), we obtain

$$
\begin{aligned}
\mathbf{E}(\Phi(S Q B E & \left.\left.S_{l \rightarrow 0}^{4, y}\right)\right) \\
& =\mathbf{E}\left(\exp \left(-\int_{0}^{y} f\left(Z_{t}^{(4)}\right) d t\right) ; \sup _{0 \leq t \leq y} Z_{t}^{(4)}<h \mid Z_{0}^{(4)}=l, Z_{y}^{(4)}=0\right) \\
& =\frac{\hat{p}^{(4)}(y ; l, 0)}{p^{(4)}(y ; l, 0)} \\
& =2 y^{2} \exp \left(\frac{l}{2 y}\right) \hat{p}^{(4)}(y ; l, 0) .
\end{aligned}
$$

Putting these expressions in (23) yields

$$
\begin{aligned}
\mathbf{E}_{0}(\exp (- & \left.V(f, \theta(T))) ; \sup _{y} L(\theta(T), y)<h\right) \\
= & 2 \alpha \int_{0}^{h}\left(\int_{0}^{\infty} \mathrm{e}^{-\alpha z} \hat{p}^{(2)}(z ; l, 0) d z\right)\left(\int_{0}^{\infty} \mathrm{e}^{-\alpha s} \hat{p}^{(4)}(s ; l, 0) d s\right) l d l \\
= & 2 \alpha \int_{0}^{h} l \hat{G}_{\alpha}^{(2, f)}(0, l) \hat{G}_{\alpha}^{(4, f)}(0, l) d l
\end{aligned}
$$

completing the proof.

Acknowledgement. We thank the anonymous referee for a very detailed report which much improved the presentation, and Olof Staffans for pointing out the role of convexity when analysing the class $\mathcal{K}$.

\section{References}

[1] M. Abramowitz and I. Stegun. Handbook of Mathematical Functions, 9th printing. Dover publications, Inc., New York, 1970. 
[2] O. Barndorff-Nielsen, P. Blaesild, and C. Halgreen. First hitting time models for generalized inverse Gaussian distribution. Stoch. Proc. Appl., 7:49-54, 1978.

[3] A.N. Borodin. Distributions of functionals of the Brownian local time, I. Th. Prob. Appl., 34(3):385-401, 1989.

[4] A.N. Borodin. On distribution of functionals of a Brownian motion stopped at the moment inverse to the range. Zapiski Nauchn. Semin. POMI (Russian), 260:50-72, 1999. English transl. in J. Math. Sciences.

[5] A.N. Borodin and P. Salminen. Handbook of Brownian Motion - Facts and Formulae, 2nd edition. Birkhäuser, Basel, Boston, Berlin, 2002.

[6] K.S. Cheng, R. Cowan, and L. Holst. The ruin problem and cover times of asymmetric random walks and Brownian motions. Adv. Appl. Prob., 32(1):177-192, 2000.

[7] L. Chosid and R. Isaac. On the range of recurrent Markov chains. Ann. Prob., 6:680-687, 1978.

[8] L. Chosid and R. Isaac. Correction to "On the range of recurrent Markov chains". Ann. Prob., 8:1000, 1980.

[9] A. Dvoretsky and P. Erdös. Some problems on random walk in space. Second Berkeley Symp. Math. Stat., pages 353-368, 1951.

[10] W. Feller. The asymtotic distribution of the range of sums of independent random variables. Ann. Math. Stat., 22:427-432, 1951.

[11] P.J. Fitzsimmons. Markov process with identical bridges. Electronic J. Prob., 3(12), 1998.

[12] P. Glynn. On the range of a regenerative sequence. Stoch. Proc. Appl., 20:105-113, 1985.

[13] I.S. Gradshteyn and I.M. Ryzhik. Table of integrals, series and products. Academic Press, New York, 1980.

[14] G. Hooghiemstra. On functionals of the adusted range process. J. Appl. Prob., 24(2):252-257, 1987. 
[15] P. Hsu and P. March. Brownian excursions from extremes. In P.A. Meyer J. Azéma and M. Yor, editors, Séminaire de Probabilités XXII, number 1321 in Springer Lecture Notes in Mathematics, pages 502-507, Berlin, Heidelberg, New York, 1988.

[16] J.-P. Imhof. On the range of Brownian motion and its inverse process. Ann. Prob., 13(3):1011-1017, 1985.

[17] J.-P. Imhof. A construction of the Brownian motion path from BES(3) pieces. Stoch. Proc. Appl., 43:345-353, 1992.

[18] N.C. Jain and S. Orey. On the range of random walk. Israel J. Math., 6:373-380, 1968.

[19] N.C. Jain and W.E. Pruitt. The range of random walk. Sixth Berkeley Symp. Math. Stat. Prob., 3:31-50, 1972.

[20] H.P. McKean, Jr. Excursions of a non-singular diffusion. Z. Wahrscheinlichkeitstheorie verw. Gebiete, 1:230-239, 1963.

[21] J. Pitman and M. Yor. Bessel processes and infinitely divisible laws. In D. Williams, editor, Stochastic Integrals, number 851 in Springer Lecture Notes in Mathematics, pages 285-370, Berlin, Heidelberg, New York, 1981.

[22] J. Pitman and M. Yor. A decomposition of Bessel bridges. $Z$. Wahrscheinlichkeitstheorie verw. Gebiete, 59:425-457, 1982.

[23] J. Pitman and M. Yor. Dilatation d'espace temps réarrangments des trajectores browniennes et quelques extensions d'une identité de Knight. C.R. Acad. Sci. Paris Serie I, 316:723-726, 1993.

[24] P. Salminen. Brownian excursions revisited. In K.L. Chung E. Cinlar and R.K. Getoor, editors, Seminar in stochastic processes, 1983, Progress in probability and statistics, Birkhauser, pages 161-188, Boston, Basel, Stuttgart, 1984.

[25] P. Salminen. One-dimensional diffusions and their exit spaces. Math. Scand., 54:209-220, 1984.

[26] P. Salminen. On last exit decomposition of linear diffusions. Studia Sci. Math. Hungar., 33:251-262, 1997. 
[27] M. Sharpe. General Theory of Markov Processes. Academic Press, San Diego, 1988.

[28] S. Tapiero and P. Vallois. Moments of the amplitude process in a random walk and approximations: computations and applications. Operat. Res., 29:1-17, 1995.

[29] B.M. Troutman. Weak convergence of the adjusted range of cumulative sums of exchangeable random variables. J. Appl. Prob., 20(2):297-304, 1983.

[30] P. Vallois. La loi gaussienne inverse généralisée comme premier ou dernier temps de passage de diffusions. Bull. Sc. math., 2e série, 115:301-368, 1991.

[31] P. Vallois. Une extension des théorèmes de Ray et Knight sur les temps locaux browniens. Prob. Th. Rel. Fields, 88:445-482, 1991.

[32] P. Vallois. Diffusion arrêtée au premier instant où l'amplitude atteint un niveau donné. Stochastics and Stochastics Reports, 43:93-115, 1993.

[33] P. Vallois. Decomposing the Brownian path via the range process. Stochastic Processes and their Applications, 55:211-226, 1995.

[34] P. Vallois. The range of a simple random walk on Z. Adv. Appl. Prob., 28:1014-1033, 1996.

[35] S. Watanabe. On time inversion of one-dimensional diffusion processes. Z. Wahrscheinlichkeitstheorie verw. Gebiete, 31:115-124, 1975.

[36] G.N. Watson. A Treatise on the Theory of Bessel Functions, 2nd edition. Cambridge University Press, London, 1948.

[37] D. Williams. Path decompositions and continuity of local time for onedimensional diffusions. Proc. London Math. Soc., 28:738-768, 1974. 